\title{
Multi-Layered Thermoelectric Power Generator
}

\author{
Ryosuke O. Suzuki ${ }^{1,2}$ \\ 1: Faculty of Engineering, Hokkaido University, \\ Kita13 Nishi8, Kita-ku, Sapporo, Hokkaido, 060-8628, Japan \\ 2: CREST researcher, Japan Science and Technology Agency, \\ Sanbancho-5, Chiyoda-ku, Tokyo 102-0075 Japan \\ rsuzuki@eng.hokudai.ac.jp
}

\section{Keywords: Thermoelectric Device, Thermal Fluid, Heat Transfer, Power Generation}

\begin{abstract}
Thermal fluids are served to give the greater $\Delta T$ to the thermoelectric (TE) junctions. For the larger power generation using a limited number of modules and the limited amounts of thermal fluids, the multi-layered TE panels can save the occupied space effectively. Because the temperatures of the fluids vary along the planer TE panels due to heat exchange through the panels, the directions of fluids is important to obtain the larger output power, $P$, from a TE power generator. The methods to stack a few long planer TE panels and to determine the fluid directions are mathematically studied from the steady-state heat exchange. $P$ for various kinds of fluid directions are calculated. $P$ commonly shows the maximum against the panel length because of the balance between the internal resistance and $\Delta T$. This maximum of $P$ can be further maximized by choosing the counter flow. The non-dimensional analysis predicts that two fluids should flow in counterwise. When two series of fluids are used, the circulation method is another key issue for the optimal TE design.
\end{abstract}

\section{Introduction}

Seebeck effect on the junctions of two different materials can generate the thermoelectric (TE) power, and the output power, $P$, depending on a temperature difference, $\Delta T$. Because we can not use the infinitively large heat bath permanently, this ideal case should be modified. It is designed that a thermal energy is extracted from the thermal bath by a fluid and passed to the TE generator. The hot and cold fluids offer $\Delta T$ between the two surfaces of the planar TE modules. The TE motive force, $E$, in the open circuit is the sum of multiplication of the relative Seebeck coefficient, $\alpha$, and the temperature difference, $\Delta \theta$, over all the serial connections. The problem to obtain the larger $P$ is therefore to give the larger $\Delta \theta$ for all the TE elements and to give the larger $\Delta T$ between the two fluids.

The heat from a hot fluid is passed to the panel surface by heat transfer, to the cold surface by heat conduction through the solid, and finally to the cold fluid again by heat transfer. The fluids are resultantly warmed or cooled along the flow path, and their temperature profile through the path, $T(x)$, changes as a function of position $x[1-9]$. This kind of problem at the steady state is known as the problem at heat exchanger through the thermal isolator.

The purpose of this work is to show the mathematical solution of the maximum power extractible from the TE panel which is heated by the hot fluid and cooled by the cold fluid. The heat transfer through the panel and the temperature change along the fluid path are analyzed mathematically.

It was expected that a compact system for the large-scale TE power generation could be realized by the multi-sheets of panel, which were efficiently given the larger $\Delta T$ by the multi-fluid paths [1,4-7]. Mathiprakasam et al. reported the precise analysis using Laplace transformation [10]. Their solution was very complex form due to Peltier effect and Joule heating although only the single sheet of panel was studied. Esarte et al. considered the temperature drop during circulation of fluid, and showed that it was fairly large [11]. The previous reports [1,2,4-8] proposed the concept to use this kind of multi-layered panels, so that the heat loss through a TE panel could be recovered by another fluid, and that the captured heat could be passed to another TE panel. It is expected that the thermal 
energy in a restricted space may become efficiently recovered by solving the given thermal and fluid conditions.

Here various kinds of TE power generation system with planer multi-panels are proposed. Applying the heat transfer analysis, analytically their temperature profiles $T(x)$ and output power, $P$, are calculated using the matrix analysis. Non-dimensional descriptions are used to select the best system, independently of the thermophysical properties of the TE materials and working fluids.

\section{Assumptions and Models}

Flat Plane Panels. It is assumed that all of the flat TE panels consist of the thermoelements with a single layer of П-type $p-n$ junctions [4]. The thermoelements are homogeneously aligned, combined tightly without open space, and connected electrically in series. The hot and cold fluids flow along the both flat surfaces of the panel, and they are isolated by the panel. However, practically there exist the electrodes connecting the thermoelements, the open space between them, the protective film between the fluid and the thermoelements, and the fins that enhance the heat exchange. Here for simplicity these effects on heat transfer are neglected, or considered as the heat transfer coefficient, $h$, at the surfaces. $h$ generally depends on the hydrodynamic behavior of the fluid and on the shape of the tube surface, but it is assumed to be constant.

This work will discuss the shape and system with the multi-planes. The number of the planes, $n$, the directions of hot and cold fluids, and the path shape for fluids are classified systematically as below.

Directions of Fluids. The fluid directions are classified, depending on the direction of cold fluid at the exist of the corresponding hot fluid: Parallel flow type (P), Counter flow type (C), Isothermal type (I) and the miXed type (X) as illustrated in Fig. 1. The isothermal type (I) is the extreme case that whole a surface of the most outside of the system is kept at a constant temperature by the infinitively large heat bath. This I type models, for example, the heat utilization of hot furnace wall such as boiling, or of huge amount of seawater as the coolant, where the surface is kept isothermally.

Path Shape. The path shapes of fluids are classified into 3 types, depending on setting of the boundary conditions: Meandering type $(\mathrm{M})$, Helical type $(\mathrm{H})$ and Branched type $(\mathrm{B})$. The meandering type (M) bends the hot fluid paths, recovers the exhaust heat from the panel to which the fluid gave the heat, and will pass the heat to the next panel. This type aims at the heat recycling and the miniaturization of the system. The helical type $(\mathrm{H})$ aims at the homogeneous $\Delta T$ for all the panels, by flowing the two fluids in counter flow. The path connection is illustrated as broken lines in Fig.1, however, the seamless three-dimensional connection can be realized in the roll pancake model [6], and in the double helical model [7]. The branched type (B) is the system that the two fluids separate into a few paths [12].

Based on the above classifications, these systems with flat panel (F) are named after the number of panel sheets $(n)$, the direction of fluid flow $(\mathrm{P}, \mathrm{C}, \mathrm{I}$ or $\mathrm{X})$ and the path shape $(\mathrm{M}, \mathrm{H}, \mathrm{B})$, as shown in Fig. 1.

\section{Equations for Output Power}

Deduction of Equations. Considering the system consisted of $n$ sheets of TE panels, a set of $n+1$ simultaneous derivative equations can be written from the heat transfers through panels,

$$
\begin{aligned}
& M_{1} C_{p, 1} d T_{1}(x) / d x= \pm K_{1} w\left(-T_{1}(x)+T_{2}(x)\right), \\
& M_{i} C_{p, i} d T_{i}(x) / d x= \pm K_{i-1} w\left(T_{i-1}(x)-T_{i}(x)\right) \pm K_{i} w\left(-T_{i}(x)+T_{i+1}(x)\right), \quad(1<i<n) \\
& M_{n+1} C_{p, n+1} d T_{n+1}(x) / d x= \pm K_{n} w\left(T_{n}(x)-T_{n+1}(x)\right),
\end{aligned}
$$

where $M_{i}, C_{p, i}, T_{i}$ are mass flow rate, heat capacity and temperature for fluid $i, w$ the width of the panel. The homogeneous temperature profile along $y$ axis is assumed inside the turbulent flow. The choice 


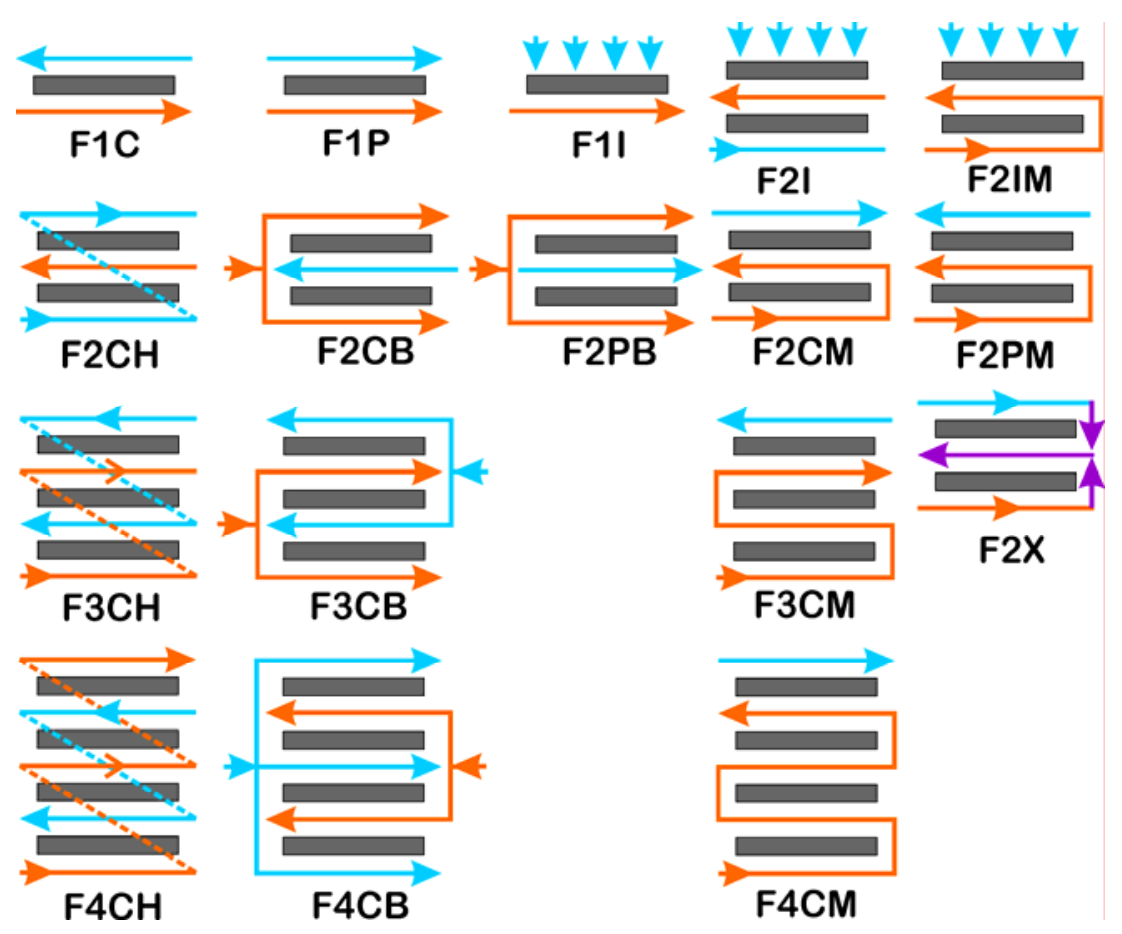

Fig.1 Analyzed fluidic systems. Flat (F) n panels are exposed in counter (C) flow, in parallel flow (P), in the meandering flow, in the isothermal flow (I) or in the mixed flow (X).

of \pm depends on the path condition. $K_{i}$ is the over-all heat transfer coefficient through the panel $i$,

$$
1 / K_{\mathrm{i}}=1 / h_{h, i}+d / \lambda+1 / h_{c, i}
$$

$h_{h, i}$ and $h_{c, i}$ are the heat transfer coefficients between hot fluid and the panel and between cold fluid and the panel, respectively, $d$ and $\lambda$ are the thickness and the average heat conductivity of the TE panel, respectively. The equation is solved for $x$ by setting the specific boundary conditions. The temperature $\theta_{i, j}(x)$ at the surface of the panel $i$ facing to the fluid $j$ is written using $T_{i}(x)$ [4-7].

$$
\theta_{i, j}(x)=T_{i}(x)-K_{i} / h_{i}\left(T_{i}(x)-T_{i+1}(x)\right) \text { and } \theta_{i, j+1}(x)=T_{i+1}(x)+K_{i} / h_{i+1}\left(T_{i}(x)-T_{i+1}(x)\right) .
$$

The electromotive force, $E$, is the summation of the temperature difference over all the panels.

$$
\begin{aligned}
& E=\sum n_{x} n_{y} \int_{0}^{w} \int_{0}^{l} S\left(\theta_{i, j}(x)-\theta_{i, j+1}(x)\right) d x d y, \\
& R_{i}=n_{x} n_{y} w l d\left(\rho_{\mathrm{p}} / s_{\mathrm{p}}+\rho_{\mathrm{n}} / s_{\mathrm{n}}\right),
\end{aligned}
$$

where $S$ is the difference of Seebeck coefficient between $\mathrm{p}$ and $\mathrm{n}$ elements, $n_{x}$ and $n_{y}$ are the number densities of TE pairs in the direction $x$ and $y$, respectively. The internal electric resistance, $R_{i}$, of the $i$-th panel is given by the specific resistivity of $\mathrm{p}$ and $\mathrm{n}$ elements, $\rho_{p}$ and $\rho_{n}$, and their cross-sectional areas, $s_{p}$ and $s_{n}$. The maximum output power, $P$, is optimized by balancing the internal and external resistance as used in normal way as

$$
P=E^{2} /\left(4 \Sigma R_{i}\right)
$$

This work uses hereafter this $P$, where the internal and external resistance are the same.

Equations for F1P system. The simultaneous equations are solved, for example, at F1P system where a single sheet of panel is exposed in parallel flow [4]. The output power $P_{1}$ is obtained in 
eq.(10). When all the terms of $M_{i} C_{p, i}$ are equivalent and all $K_{i}$ are equal to a constant $K$, the output power $P_{2}$ is obtained in the simpler form as eq. (11),

$$
\begin{aligned}
& P_{1}=\left(M_{h} C_{p, h} M_{c} C_{p, c} S\right)^{2} n_{x} n_{y} d\left(T_{h}^{i n}-T_{c}^{i n}\right)^{2}\left(1-e^{-D K w l}\right)^{2} /\left(4 w l\left(M_{h} C_{p, h}+M_{c} C_{p, c}\right)^{2}\left(\rho_{\mathrm{p}} / s_{\mathrm{p}}+\rho_{\mathrm{n}} / s_{\mathrm{n}}\right) \lambda^{2}\right), \\
& P_{2}=M C_{p}(K d / \lambda) Z\left(T_{h}^{i n}-T_{c}^{i n}\right)^{2} L\left(s_{\mathrm{p}}, s_{\mathrm{n}}\right) \Phi_{1 \mathrm{P}}(X),
\end{aligned}
$$

where $Z$ is the figure of merit, known as the material parameter, and $L\left(s_{p}, s_{n}\right)$ is the characteristic function of the cross-sectional area, and it is optimized to be its maximum as 1 when $\left(s_{n} / s_{p}\right)^{2}=$ $\rho_{\mathrm{n}} \lambda_{\mathrm{p}} / \rho_{\mathrm{p}} \lambda_{\mathrm{n}} \cdot \Phi_{1 \mathrm{P}}(\mathrm{X})$ is a non-dimensional function depending on the fluidic patterns. $X$ is the non-dimensional length of the system, defined as $X=K w l / M C_{p}$.

$$
\Phi_{1 \mathrm{P}}(X)=\left(1-e^{-2 X}\right)^{2} /(16 X) \text {. }
$$

Its maximum, $\Phi_{1 \mathrm{P}}^{\max }$, is $5.091 \times 10^{-2}$ when $X$ satisfies as $4 X+1=\mathrm{e}^{2 X}$. This optimized length $X_{\mathrm{opt}}$ can be numerically solved as $X_{\mathrm{opt}}=0.6282$. When $L\left(s_{p}, s_{n}\right)=1$ and $\Phi_{\mathrm{F} 1 \mathrm{P}}(X)=\Phi_{\mathrm{F} 1 \mathrm{P}}\left(X_{\mathrm{opt}}\right)=\Phi_{\mathrm{F} 1 \mathrm{P}}{ }^{\max }, P_{2}$ depends only on the mass flow through $M, h_{h}$ and $h_{c}$, as well as $Z$ and the square of input temperature difference, $T_{h}{ }^{i n}-T_{c}{ }^{i n}$. $\Phi_{\mathrm{F} 1 \mathrm{P}}(X)$ is determined only by the system design but independent of the materials and fluid properties. Therefore, $\Phi_{\mathrm{F} 1 P}{ }^{\max }$ is defined as the system parameter, and $X_{\mathrm{opt}}=$ 0.6282 as the characteristic length at F1P system.

The same procedures could be applied for all the systems shown in Fig.1, and it was found that the output power $P_{2}$ can be commonly obtained as the form of eq.(11), when the non-dimensional function $\Phi(X)$ is defined adjusting to the individual system design. The function $\Phi(X)$ is characteristic

\begin{tabular}{|c|c|}
\hline System & $\Phi(\mathrm{X})$ \\
\hline F1P & $\Phi(X)=\frac{\left(1-e^{-2 X}\right)^{2}}{16 X}$ \\
\hline $\mathrm{F} 1 \mathrm{C}$ & $\Phi(X)=\frac{X}{4(1+X)^{2}}$ \\
\hline F1I & $\Phi(X)=\frac{\left(1-e^{-X}\right)^{2}}{4 X}$ \\
\hline F2PM & $\Phi(X)=\frac{\left\{-1+\sqrt{2}-(1+\sqrt{2}) e^{2 \sqrt{2} X}+2 e^{(1+\sqrt{2}) X}\right\}^{2}}{2\left\{2-\sqrt{2}+(2+\sqrt{2}) e^{2 \sqrt{2} X}\right\}^{2} X}$ \\
\hline $\mathrm{F} 2 \mathrm{CB}$ & $\Phi(X)=\frac{X}{2(1+2 X)^{2}}$ \\
\hline $\mathrm{F} 2 \mathrm{~PB}$ & $\Phi(X)=\frac{\left(1-e^{-4 X}\right)^{2}}{32 X}$ \\
\hline $\mathrm{F} 2 \mathrm{CM}$ & $\Phi(X)=\frac{\left(1-e^{-X}\right)^{2}}{8 X}$ \\
\hline $\mathrm{F} 2 \mathrm{CH}$ & $\Phi(X)=\frac{1}{2 X(2+\operatorname{coth} X)^{2}}$ \\
\hline F2IM & $\Phi(X)=\frac{\left\{-\sqrt{5} e^{\frac{X}{2}}+\sqrt{5} \cosh \left(\frac{\sqrt{5} X}{2}\right)+3 \sinh \left(\frac{\sqrt{5} X}{2}\right)\right\}^{2}}{8 X\{2+3 \cosh (\sqrt{5} X)+\sqrt{5} \sinh (\sqrt{5} X)\}}$ \\
\hline
\end{tabular}
for each system and listed in Table 1.

Table 1 Calculated non-dimensional output power $\Phi(\mathrm{X})$. 


\begin{tabular}{|c|c|}
\hline F2I & $\Phi(X)=\frac{e^{(-1-\sqrt{5}) X}\left\{(-3+\sqrt{5}) e^{\frac{X}{2}}-2 \sqrt{5} e^{\frac{\sqrt{5} X}{2}}+(3+\sqrt{5}) e^{\left(\frac{1}{2}+\sqrt{5}\right) X}\right\}^{2}}{32 X\{-2+7 \cosh (\sqrt{5} X)+3 \sqrt{5} \sinh (\sqrt{5} X)\}}$ \\
\hline $\mathrm{F} 2 \mathrm{X}$ & $\Phi(X)=\frac{\left(1-e^{-X}\right)^{2}}{8 X}$ \\
\hline $\mathrm{F} 3 \mathrm{CM}$ & $\Phi(X)=\frac{\{-1+(1+2 X) \cosh (\sqrt{2} X)\}^{2}}{6 X\{\sqrt{2}(2+X) \cosh (\sqrt{2} X)+\sinh (\sqrt{2} X)\}^{2}}$ \\
\hline $\mathrm{F} 3 \mathrm{CH}$ & $\Phi(X)=\frac{\left\{2 X \cosh \left(\frac{X}{\sqrt{2}}\right)+\sqrt{2} \sinh \left(\frac{X}{\sqrt{2}}\right)\right\}^{2}}{3 X\left\{(2+4 X) \cosh \left(\frac{X}{\sqrt{2}}\right)+\sqrt{2}(2+X) \sinh \left(\frac{X}{\sqrt{2}}\right)\right\}^{2}}$ \\
\hline $\mathrm{F} 3 \mathrm{CB}$ & $\Phi(X)=\frac{[\sqrt{2}\{-1+(1+4 X) \cosh (2 \sqrt{2} X)\}+(1+6 X) \sinh (2 \sqrt{2} X)]^{2}}{12 X[\sqrt{2}\{-1+(3+4 X) \cosh (2 \sqrt{2} X)\}+2(2+3 X) \sinh (2 \sqrt{2} X)]^{2}}$ \\
\hline $\mathrm{F} 4 \mathrm{CM}$ & 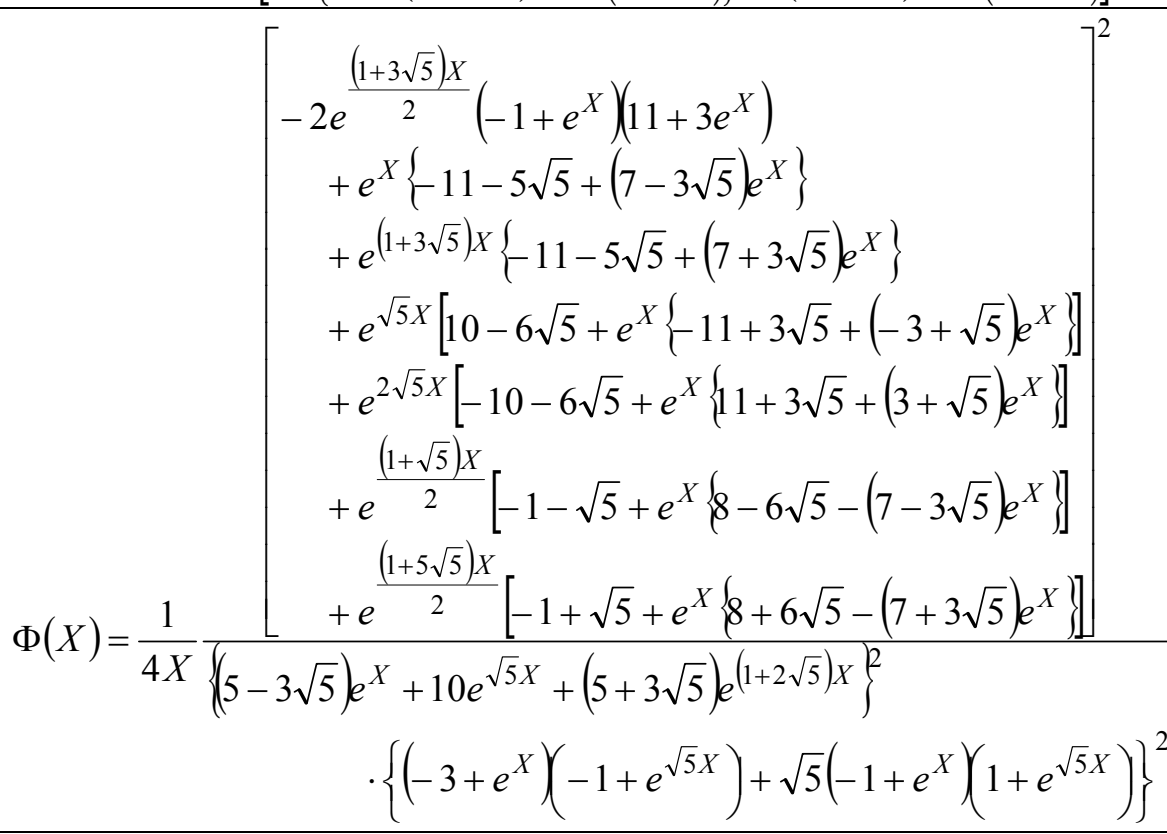 \\
\hline $\mathrm{F} 4 \mathrm{CH}$ & 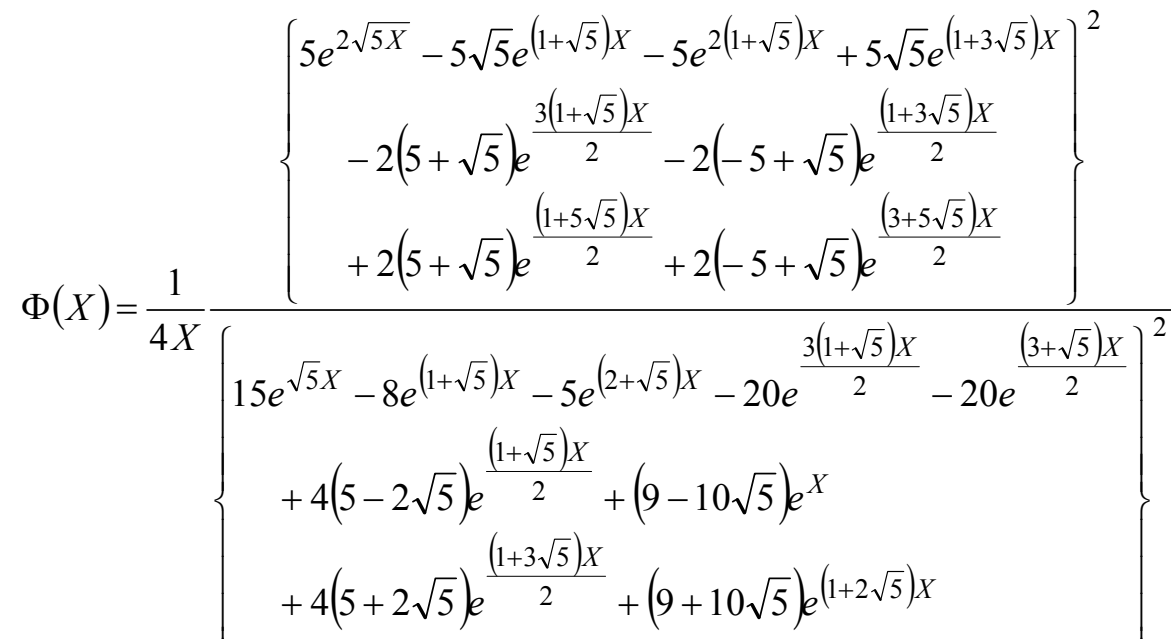 \\
\hline
\end{tabular}




\begin{tabular}{|l|l|}
\hline F4CB & $\Phi(X)=\frac{\left\{-1+e^{5 X}(1+45 X)\right\}^{2}}{4 X\left\{2-9 e^{5 X}(3+10 X)\right\}^{2}}$ \\
\hline
\end{tabular}

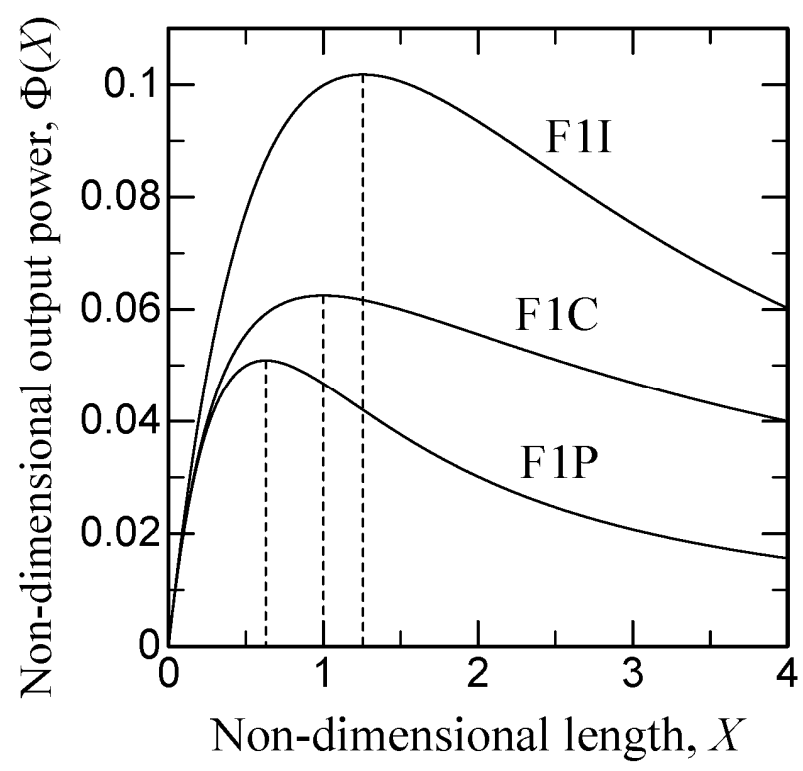

Fig.2 Calculated non-dimensional output power for the flat-one- panel (F1) systems.

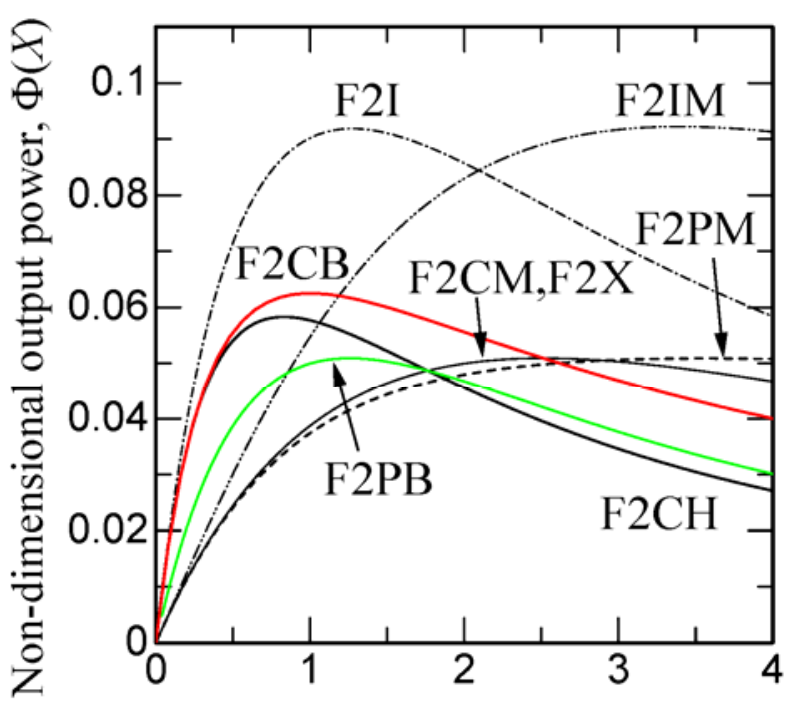

Total non-dimensional length, $\Sigma X$

Fig.3 Calculated non-dimensional output power for the flat-two-panels (F2) systems.

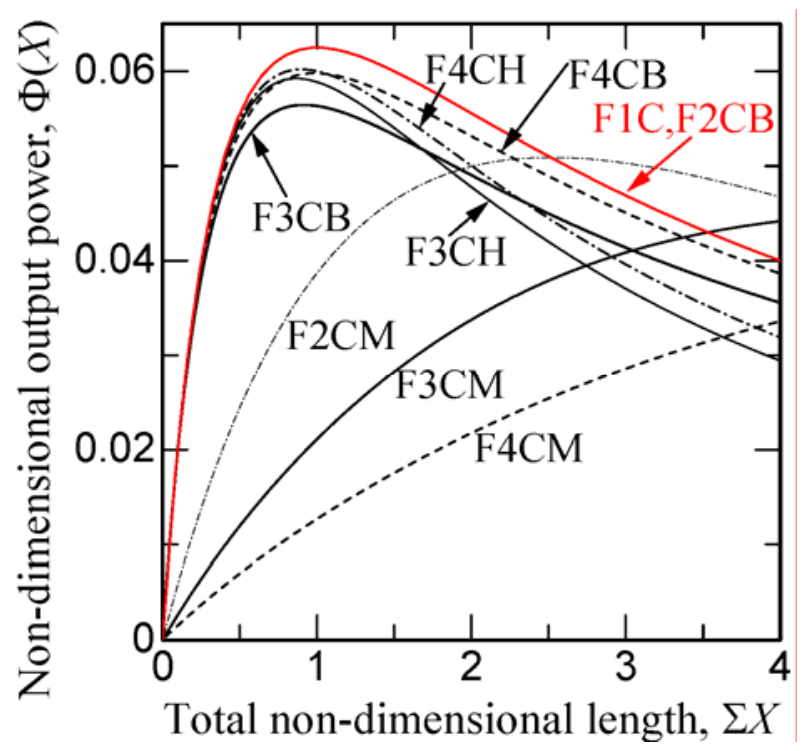

Fig.4 Calculated non-dimensional output power for the flat-multi-panels (F3,F4) systems.

Fig.2, 3 and 4 show the $X$ dependency of $\Phi(X)$. In case of two-panel and three panel systems, the summations of $X$ for all the panels are taken as the horizontal axis in Fig. 3 and 4. This summation of $X$ corresponds to the total length of the panel necessary for the system construction. 


\section{Discussions}

$\Phi_{\mathrm{F} 1 \mathrm{II}}(X)_{\max }, \Phi_{\mathrm{F} 2 \mathrm{I}}(X)_{\max }$, and $\Phi_{\mathrm{F} 2 \mathrm{IM}}(X)_{\max }$ are significantly high, because a cold surface is kept at a constant temperature for these systems. It was basically assumed in the isothermal type (I) that the infinitive fast heat supply and heat compensation was available to keep a constant temperature. This assumption was mathematically realized that a limit of F1P and F1C system was F1I system as they approached to the infinitive. The infinitive fast cooling in I type is the reason of high power.

Secondly, the power of F1C is larger than that of F1P, while the characteristic length of F1C is longer. As shown in Fig.2, $\Phi_{\mathrm{F} 1 \mathrm{C}}(X)$ is larger than $\Phi_{\mathrm{F} 1 \mathrm{P}}(X)$ for all $X$. The tendency that the counter flow can generate larger power than the parallel flow is valid also in two sheets of panels: $\Phi_{\mathrm{F} 2 \mathrm{CM}}(X)>$ $\Phi_{\mathrm{F} 2 \mathrm{PM}}(X)$.

When we compare by the path shapes $(\mathrm{H}, \mathrm{B}$ and $\mathrm{M})$, the helical type $(\mathrm{H})$ has the highest $\Phi$ and the shortest length $X_{\text {opt }}$. The branched type (B) in 3 and 4 panels systems have a little smaller $\Phi$ and a little longer $X_{\mathrm{opt}}$ than those of $\mathrm{H}$ type. The geometrical construction of $\mathrm{H}$ type seems possible as reported previously, although the total path length for fluid becomes longer and it causes larger pressure loss.

In the branched fluids, the heat exchange at the outer channel becomes important, because the outer panel (wall of the system) is considered as the adiabatic barrier. It is noted that $\Phi_{\mathrm{F} 1 \mathrm{C}}(X)=$ $\Phi_{\mathrm{F} 2 \mathrm{CB}}(\Sigma X)$ as shown in Fig. 3 when the fluid is branched in an equal flow rate. If the fluid can be branched in an optimal ratio, $\Phi_{\mathrm{F} 3 \mathrm{CB}}(\Sigma X)$ and $\Phi_{\mathrm{F} 4 \mathrm{CB}}(\Sigma X)$ in Fig. 3 increases to $\Phi_{\mathrm{F} 1 \mathrm{C}}(X)[12]$. The flow rate control is critical in the branched type.

As the number of sheets of panels increases for $\mathrm{H}$ and B types, their output power increase and their optimal lengths $X_{\text {opt }}$ become shorter. In these types, the multi-panel construction thus improves the thermoelectric properties. However, the output power decreases for Meandering (M) types. M type needs the longest $X_{\mathrm{opt}}$ in these three types because the heat exchange between the hot and cold fluids is not effective. Namely, the heat recycling by $M$ type needs plenty of thermo-elements.

Except for isothermal types, the highest output power is obtained in F1C and F2CB systems. $\Phi^{\max }$ becomes gradually smaller at $\mathrm{F} 4 \mathrm{CH}, \mathrm{F} 4 \mathrm{CB}, \mathrm{F} 3 \mathrm{CH}, \mathrm{F} 2 \mathrm{CH}, \mathrm{F} 3 \mathrm{CB}$ systems in this order. $\Phi^{\max }$ for the multi-panels systems gets closer to $\Phi_{\mathrm{F} 1 \mathrm{C}}{ }^{\mathrm{max}}$, as the number of sheets increases in $\mathrm{H}$ and $\mathrm{B}$ types. This is because the effect of non-heat exchange through the two adiabatic walls decreases and the effective heat exchange among the fluids occurs, when many panels are used.

The shortest length is achieved at $\mathrm{F} 4 \mathrm{CH}$ system, where the difference with output of F1C system is only $3.71 \%$ and the length of $\mathrm{F} 4 \mathrm{CH}$ is $10.0 \%$ shorter. Therefore, the output per unit length of panel is higher for $\mathrm{H}$ types than that of $\mathrm{F} 1 \mathrm{C}$ system.

Although the non-dimensional functions $\Phi(X)$ are calculated as a function of length $X$ here, it is also a function of the panel width $w$. This can be easily analyzed and confirmed using the similar procedure concerning $w$. Therefore, $X$ might be reffered as the non-dimensional area of the panel. This is verified by the fact that the non-dimensional length $X$ consists of the product $w l$. The unique maxmim in $\Phi(X)$ shows that the optimum surface area of TE panel is defined by $X_{\text {opt }}$.

In summary, the multiplication of panels decreases the output a little, but it can decrease the surface of thermoelectric power generator. Namely, the power generation with the higher material efficiency become possible by stacking the panels and by flowing the fluids in counter.

\section{Conclusions}

Thermal fluids can give the greater $\Delta T$ between the TE panel surfaces. For the larger power generation using a limited number of modules and the limited amounts of thermal fluids, the multi-layered TE panels can save the occupied space effectively. Because the temperature profiles $T_{i}(x)$ of the fluids vary along the planer TE panels due to heat exchange through the panels, the directions of fluids is important to obtain the larger output power, $P$, from a TE power generator. The methods to stack a few long planer TE panels and to determine the fluid directions are mathematically studied from the steady-state assumption in heat exchange. 
$P$ for various kinds of flow conditions are calculated. $P$ commonly shows the maximum against the panel length because of the balance between the internal resistance and $\Delta T$. This maximum of $P$ can be further maximized by choosing the counter flow. Except for ideal heating and cooling, the non-dimensional analysis predicts that two fluids should flow in counter. When two series of fluids are used, F1C and F2CB systems were the optimal TE design.

\section{Acknowledgements}

The author thanks to Mr. Daisuke Tanaka at Kyoto University and Mr. Yuto Sasaki at Hokkaido University for their calculations. This work was financially supported in part by Japan Science and Technology Agency (JST) - Core Research of Evolutional Science \& Technology (CREST) program.

\section{References}

[1] K. Ono and R. O. Suzuki, Proc. 17th Intern. Conf. on Thermoelectrics, IEEE, Piscataway, NJ, (1998), p.515-518.

[2] D.M. Rowe and M. Gao, J. Power Sources, Vol.73 (1998) p.193-198.

[3] T. Kyono, R. O. Suzuki and K. Ono, IEEE Transactions on Energy Conversion, Vol.18 [2] (2003) p.330-334.

[4] R. O. Suzuki and D. Tanaka, J. Power Sources, Vol.122 [2] (2003) p.201-209.

[5] R. O. Suzuki and D. Tanaka, J. Power Sources, Vol.124 [1] (2003) p. 293-298.

[6] R. O. Suzuki and D. Tanaka, J. Power Sources, Vol.132 [1-2] (2004) p.266-274.

[7] R. O. Suzuki, J. Power Sources, Vol.133 [2] (2004) p.277-285.

[8] C. Lertsatitthanakorn, N. Khasee, S. Atthajariyakul, S. Soponronnarit, A. Therdyothin, R. O. Suzuki, Solar Energy Materials \& Solar Cells, Vol.92 [9] (2008) p.1105-1109.

[9] J.Yu and H. Zhao, J. Power Sources, Vol.172 [1-2] (2007) p.428-434.

[10]B. Mathiprakasam, T. Sutikno and J. Beeson, in Proc. 4th Intern. Conf. on Thermoelectrics, IEEE, Piscataway, NJ, (1982), p.61.

[11] J. Esarte, G. Min and D.M. Rowe, J. Power Sources, Vol.93 (2001) p.72-76.

[12]R. O. Suzuki and Y. Sasaki, submitted to J. Electronic Materials (2009). 